\title{
El Grupo Mesoamericano de Ciencias Médicas
}

Mesoamérica, es la región del continente americano que comprende la mitad meridional de México y los territorios de Guatemala, El Salvador, Belice, el occidente de Honduras, Nicaragua y Costa Rica. Es un área definida por la cultura en la que se desarrolló una civilización indígena dentro del marco de un mosaico de gran diversidad étnica y lingüística.

Las fronteras de Mesoamérica estaban delimitadas, al norte, por el río Sinaloa y las cuencas de Lerma y Soto de la Marina en México y, al sur, por el río Ulúa (Honduras), los grandes Lagos de Xolotlán y Cocibolca (Nicaragua) y la Península de Nicoya, en Costa Rica. Dicha área incluyó algunas de las culturas más avanzadas de las Américas, como la olmeca, zapoteca, teotihuacana, maya, mixteca, totonaca y azteca, entre otros.

En 1943, durante un congreso sobre América, Paul Kirchoff, etnógrafo alemán, presentó el concepto de Mesoamérica, delimitando el área geográfica y propuso una serie de características que definían a las culturas de la región: la organización social patrilineal, pirámides escalonadas, juegos de pelota y palacios alrededor de plazas con pisos de estuco, uso de dos calendarios, uno ritual de 260 días (tzolkin), y otro solar (haab) de 365 días. Asimismo, la numeración con base veinte y la escritura pictográfica-jeroglífica, sacrificios humanos como parte de expresiones religiosas, el culto a ciertas divinidades -entre las que sobresalen las divinidades del agua, el fuego y la Serpiente Emplumada- y la ausencia de metalurgia, entre otros elementos, compartidos por los pueblos de la región Mesoamericana precolombina (1).

Desde 2005 se inicia un programa de intercambio entre académicos del Centro de Investigaciones Regionales "Dr. Hideyo
Noguchi" de la Universidad Autónoma de Yucatán (CIR-HN) y el Centro de Investigaciones en Hematología y Trastornos Afines de la Universidad de Costa Rica (CIHATA-UCR). En ese año viajaron a San José Costa Rica, tres académicos del CIR-HN para efectuar estancias de investigación con énfasis en métodos de biología molecular para el diagnóstico de hemopatías y enfermedades metabólicas. En el año 2011, el CIR-HN organizó un curso de introducción a la Biología Molecular y fueron profesores titulares del curso dos académicos del CIHATA-UCR.

En el curso de 2012, ya habiéndose integrado un grupo de la UNAN-León de Nicaragua, se somete a la convocatoria ANUIESCSUCA 2012, la propuesta de investigación conjunta Variantes genéticas: Inserción/deleción del gen de la Enzima Convertidora de Angiotensina, C677T del gen de la Metiltetrahidrofolato Reductasa y del gen CCR5 delta 32, asociados con el desarrollo de la nefropatía en pacientes con Diabetes Mellitus tipo 2 de áreas urbanas de San José Costa Rica, León de Nicaragua y Yucatán, México. Este trabajo resultó aprobado, lo que permitió que en octubre de 2012 y enero de 2013 estuvieran en el Laboratorio de Hematología del CIR-HN, representantes de los 3 países. Fue precisamente durante ese tiempo que se define la intención de formar un grupo de Investigación multidisciplinario y multinacional, ya que al compartir desde la era precolombina una serie de características que definían a las culturas de la región y que eran comunes por todos los pueblos de Mesoamérica era posible también encontrar temáticas, problemas y oportunidades de investigación de situaciones comunes de salud en la región, así como el apoyo a los programas de posgrado de los diferentes países.

Es este contexto histórico-cultural 
González-Martínez et al.

y académico el que aglutina a un grupo de investigadores del campo de la salud, académicos y clínicos de Costa Rica, Nicaragua, Panamá, Honduras y México, y se conforma oficialmente desde el año 2013, "El Grupo Mesoamericano de Ciencias Médicas". Este grupo de trabajo académico surge a partir del I Congreso Mesoamericano de Ciencias Médicas celebrado en San Jose de Costa Rica, auspiciado por Centro de Investigaciones Regionales "Dr Hideyo Noguchi” de la Universidad Autónoma de Yucatán, Mérida-México y el Centro de Investigación en Hematología y Trastornos afines de la Universidad de Costa Rica, en conjunto con el Departamento de Histoembriología, de la UNAN-León, Nicaragua y de profesores de Alemania y Hungría.

En el año 2014, se realizó el II Congreso Mesoamericano de Ciencias Médicas, con la participación de invitados de Venezuela y Alemania, además de los fundadores. Esta actividad científica contó con el apoyo de la Universidad Nacional Autónoma Nicaragua, en León, y con la participación de cerca de 80 investigadores y docentes de las instituciones mencionadas. Dentro del marco de este Congreso, se creó el premio del Investigador del Grupo Mesoamericano, que busca reconocer a quien se distingue por fortalecer en la región, la investigación científica en el área de salud. En este II Congreso, fue designado Pedro González Martínez, especialista de la UADY como nuevo Presidente del Grupo Mesoamericano de Ciencias Médicas.

El grupo cuenta con el apoyo de expertos de renombre internacional, en especial de Universidades e institutos Europeos como el Instituto de Hematología Experimental y Medicina Transfusional, de la Universidad Clinica de Bonn y la Universidad Friedrich Schiller de Jena, ambas de Alemania; el Instituto de Salud Publica en Genómica de la Universidad de Maastricht, Holanda, Departamento de
Biología Molecular y Genética de la Universidad de Aarhus de Dinamarca y la Universidad de Debrecen, Hungría, quienes han aportado su conocimiento y experiencia sobre diversos temas, a las actividades científicas que se desarrollaron en Costa Rica y Nicaragua.

El principal objetivo del grupo es fortalecer entre sus miembros la investigación científica en el área de salud en temas de interés para la región mesoamericana, por medio de investigaciones conjuntas, apoyo de pasantías y actividades científicas de capacitación, e investigaciones epidemiológicas y sociales que permitan proponer guías de salud para los países miembros. Los objetivos primarios del grupo consideran mejorar la investigación en los programas de posgrado, la consecución de fondos en los diferentes países para realizar investigaciones de interés regional, en temas que se detecten y consideren prioritarios para nuestras poblaciones. Se debe recalcar que la multidisciplinaridad de sus miembros fortalece, aún más, el proceso de investigación, pues se cuenta con profesionales de diversas áreas agrupados en equipos de académicos e investigadores. Todo esto, con el fin de conformar un grupo consolidado con proyección tanto en la docencia e investigación en busca de la excelencia académica y que cuente con el apoyo de expertos

Actualmente, el grupo cuenta con más de 50 miembros de diversos países de Centroamérica, México y Venezuela y se ha programado para que del 27 al 30 de octubre del año en curso, en Mérida, Yucatán se efectúe el III Congreso Mesoamericano, con la visión de ampliar horizontes, y consolidar grupos de trabajos en las áreas de interés, identificadas en las actividades previas. Las sedes del congreso en Mérida serán el Centro de Investigaciones Regionales "Dr. Hideyo Noguchi" y el edificio central de la UADY y se contará con la participación de investigadores y académicos de Costa Rica, Nicaragua, Honduras, Panamá, Venezuela,

\section{Revista Biomédica}


El Grupo Mesoaméricano de Ciencias Médicas

Alemania y México. Por ello, convocamos a los investigadores, clínicos y docentes de la región a integrarse al Grupo Mesoamericano y a participar activamente en el III Congreso en Mérida.

Dr. Pedro González Martínez.

Dra. Ligia Vera Gamboa.

Centro de Investigaciones Regionales-HN.

Universidad Autónoma de Yucatán.

Dra. Lizbeth Salazar Sánchez

CIHATA-Escuela de Medicina.

Universidad de Costa Rica.

\section{REFERENCIAS}

1. Kirchhoff, Paul. Mesoamérica, sus límites geográficos, composición étnica y caracteres culturales. [en línea]. Xalapa Ver., AL FIN LIEBRE EDICIONES DIGITALES, 2009. 12 pp [ref. 9 de marzo de 2015]. Disponible en Web: http://.alfinliebre.blogspot.com/. 